\title{
Overexpression of SMARCA2 or CAMK2D is associated with cisplatin resistance in human epithelial ovarian cancer
}

\author{
XIAOLI XU ${ }^{1 *}$, ZHIGUO ZHENG $^{2 *}$, LANLAN JIA ${ }^{1}$, SHASHA SUO ${ }^{1}$, BOWEN LIU ${ }^{1}$, \\ TIANNING SHAO ${ }^{1}$, QINQING TU ${ }^{1}$, YUEJIN HUA ${ }^{1}$ and HONG XU ${ }^{1}$ \\ ${ }^{1}$ Institute of Nuclear-Agricultural Sciences, Zhejiang University, Hangzhou, Zhejiang 310029; \\ ${ }^{2}$ Institute of Zhejiang Cancer Research, Zhejiang Cancer Hospital, Hangzhou, Zhejiang 310022, P.R. China
}

Received October 27, 2017; Accepted June 13, 2018

DOI: $10.3892 / 01.2018 .9109$

\begin{abstract}
Ovarian cancer is one of the most lethal types of gynecological cancer. Drug resistance is a major underlying cause of treatment failure, which has lead to continued poor mortality and morbidity rates in patients. In the present study, highly sensitive transcriptome sequencing was performed to systematically identify differentially expressed mRNAs in cisplatin-sensitive (A2780) and -resistant (A2780-DR) cells. Calcium/calmodulin dependent protein kinase IID (CAMK2D) and SWI/SNF related matrix associated actin dependent regulator of chromatin subfamily A member 2 (SMARCA2) were identified as exhibiting increased expression in cisplatin-resistant cells. Overexpression of either SMARCA2 or $C A M K 2 D$ led to a significant increase in the survival rates of A2780 and SKVO3 cells following cisplatin treatment. To further verify the contribution of these two genes in the development of drug resistance, the RNA levels in tissues with different recurrence-free survival (RFS) rates were compared. An increased mRNA level of CAMK2D was detected in samples with shorter RFS rates. An apoptosis assay revealed that overexpression of SMARCA2 or CAMK2D increased the resistance of ovarian cancer cells to cisplatin, as indicated by the decreased apoptotic cell populations. The levels of these two genes also affected the cell cycle and apoptosis-associated protein expression. Quantitative proteomic analyses revealed that overexpression of $S M A R C A 2$ or $C A M K 2 D$ influences
\end{abstract}

Correspondence to: Dr Hong Xu, Institute of Nuclear-Agricultural Sciences, Zhejiang University, 268 Kaixuan Road, Hangzhou, Zhejiang 310029, P.R. China

E-mail: xuhong1685@163.com

*Contributed equally

Abbreviations: DDP, dichlorodiamine platinum (cisplatin); DR, drug resistance; RFS, recurrence free survival

Key words: cisplatin resistance, transcriptome sequencing, ovarian cancer, SWI/SNF related matrix associated actin dependent regulator of chromatin subfamily A member 2, calcium/calmodulin dependent protein kinase II $\delta$, quantitative proteomics multiple metabolism and cancer-associated signaling pathways, which are critical for responses to cisplatin treatment and drug resistance development.

\section{Introduction}

Ovarian cancer, one of the most common gynecologic malignancies and the fifth leading cause of cancer-related death in women worldwide (1), has the highest mortality rate among all gynecologic malignancies with limited efficiency of treatment due to poorly defined etiological factors (2). Platinum-based chemotherapy (in combination with paclitaxel or docetaxel) is the first-line treatment for advanced disease (3). However, the survival rates of ovarian cancer patients are significantly lower in cases that develop resistance to platinum chemotherapy (4). Determination of the mechanisms underlying drug resistance in ovarian cancer is therefore an urgent medical requirement. Accumulating studies have found that the expression levels of different RNAs, including mRNAs, lincRNAs and miRNAs, have a strong relationship with cancer development and treatment recovery (5). For example, MDR1 overexpression through promoter fusion is reported to contribute to acquired resistance in high-grade serous ovarian cancer (HGSC) (6). HMGAl in adherent ovarian cancer cells increases resistance to chemotherapeutic agents (7). lincRNA H19 is involved in tumor development, progression and metastasis (8). A number of miRNAs, including miR-23a (9), miR-27a (5,6), miR-106a (3), miR-133a (4), miR-145 (10), miR-181b (11), miR-218 (3) and miR-326 (4), appear to be involved in the development of drug resistance by regulating relative gene expression.

Recent analyses have showed that NRF1, MTERF, and $C A M K 2 D$ are strongly associated with EOC (epithelial ovarian cancer) risk $(12,13)$, dysregulated in many malignancies, and participate in cell growth, apoptosis and angiogenesis (14).Application of calcium/calmodulin dependent protein kinase II delta $(C A M K 2 D$; rs10023113) as a potential prognostic marker for overall survival of early-stage NSCLC in Chinese populations $(15,16)$. On the other hand, SWI/SNF-related matrix-associated actin-dependent regulator of chromatin, subfamily a, member 2 (SMARCA2) and SWI/SNF subunits have been frequently characterized as tumor suppressors, with $20 \%$ cancers bearing mutations in these genes (17-19).Although considerable research attention 
has focused on ovarian cancer development, limited reports have documented the mechanisms by which these gene products influence drug resistance in cisplatin-resistant and -sensitive cells. Large-scale studies evaluating the association of different gene products with cisplatin resistance in ovarian cancer cells are warranted to improve therapeutic options in the clinic.

In the present study, we examined the overall mRNA expression profiles of cisplatin-sensitive and -resistant A2780 cells, and verified the different expression patterns using three specific cell lines and patient tissue samples. Quantitative proteomic analyses of SMARCA2 and CAMK2D overexpressed individually in A2780 cells provided evidence of association of both genes with development of drug resistance mainly through RNA and energy metabolism.

\section{Materials and methods}

Patient and sample data. HGSC tissues with different RFS were supplied by the Zhejiang Cancer Hospital Biospecimen Repository. The present study was approved by the Zhejiang Cancer Hospital Ethics Committee (2012-03-7) and informed consent was obtained from all participants prior to their inclusion. Histopathology and tumor grade were evaluated. Tumor stage was determined by a gynecologic oncologist based on FIGO classification criteria. All procedures were carried out in accordance with the approved guidelines of Zhejiang Cancer Research Institution.

Next-generation sequencing and bioinformatics analysis. Total RNA (without ribosomal RNA) of cisplatin-sensitive and -resistant A2780 cells was extracted and submitted for sequencing. The detailed procedures are described in previous reports (5). A gene was considered significantly different at fold change of $>2$ and P-value $<0.001$. Gene Ontology (GO) annotation was performed using the UniProt-GOA database (http://www.ebi.ac.uk/GOA). Kyoto Encyclopedia of Genes and Genomes (KEGG) pathways were annotated using the online KEGG Automatic Annotation system. A similar approach was used for proteomics data analysis.

Reverse transcription-quantitative polymerase chain reaction analysis (RT-qPCR). Total RNA was extracted from cultured cells with TRIzol reagent (Invitrogen; Thermo Fisher Scientific, Inc., Waltham, MA, USA) and1 $\mu \mathrm{g}$ used for cDNA synthesis with a PrimeScript RT reagent kit (Takara Biotechnology Co., Ltd., Dalian, China). Amplification and melting curve analyses were performed using a LightCycler 480 RT-qPCR system and LightCycler 480 SYBR-Green I Master Mix (Roche Applied Science, Mannheim, Germany). Amplification cycles were $95^{\circ} \mathrm{C}$ for $3 \mathrm{~min}, 35$ cycles at $95^{\circ} \mathrm{C}$ for $30 \mathrm{sec}, 55^{\circ} \mathrm{C}$ for $30 \mathrm{sec}, 72^{\circ} \mathrm{C}$ for $30 \mathrm{sec}$, followed by $72^{\circ} \mathrm{C}$ for $3 \mathrm{~min}$. $\beta$-Actin was used for normalization and the relative levels calculated using the $2^{-\Delta \Delta \mathrm{Ct}}$ method. Tissue samples were obtained from patients with different recurrence-free survival (RFS) rates (RFS $>12$ months classified as the cisplatin-sensitive group and RFS $\leq 12$ months as the cisplatin-resistant group), and cDNA hybrids of 32 samples were mixed as the control. Each PCR reaction was performed using one premixed reference template. All reactions were conducted in triplicate.
Cell culture and plasmid transfection. A2780 and SKOV3 cells were purchased from Cobioer (Nanjing, China) and verified by Shanghai Biowing Applied Biotechnology Co., Ltd. Expression plasmids containing CAMK2D (NM_172115) and SMARCA2 (NM_003070) were purchased from Shanghai GeneChem Co., Ltd., (Shanghai, China) and Lipofect2000 ${ }^{\mathrm{TM}}$ obtained from Invitrogen; Thermo Fisher Scientific, Inc.

Cisplatin-sensitive A2780 and SKOV3 cells were seeded into six-well plates and allowed to reach $90-95 \%$ confluence at $37^{\circ} \mathrm{C}$ before transfection. Cells were transfected with expression plasmids containing CAMK2D or SMARCA2 and those containing empty vector used as a control. The ratio of plasmid to Lipofect2000 was 1:2. After $6 \mathrm{~h}$ transfection, the medium was replaced with complete culture medium. Cells were continuously cultured until harvesting for analysis after $48 \mathrm{~h}$ transfection.

Cell viability assay. Cisplatin-sensitive A2780 cells transfected with different plasmids were seeded in 96-well plates at a density of $10^{4}$ cells per well and cultured at $37^{\circ} \mathrm{C}$ in $5 \%$ $\mathrm{CO}_{2}$. After $24 \mathrm{~h}$ culture, cells in each well were incubated with cisplatin at various concentrations for $24 \mathrm{~h}$. The drug was removed by replacing with DMEM containing $20 \mu \mathrm{l}$ MTS (Promega Corporation, Madison, WI, USA) for every $100 \mu \mathrm{l}$ culture medium in each well. Cells were further incubated for $4 \mathrm{~h}$ at $37^{\circ} \mathrm{C}$ before the assay. Absorbance was measured using an automated microplate reader (SpectraMax M5) at $490 \mathrm{~nm}$ and all reactions were carried out in triplicate.

Cell apoptosis and cell cycle analysis. Cells were transfected with expression plasmids containing CAMK2D, SMARCA2 or empty vector, incubated for $48 \mathrm{~h}$ and treated with $10 \mu \mathrm{M}$ cisplatin. At $24 \mathrm{~h}$ after treatment, the medium was removed and $1 \times 10^{6}$ cells collected in $1.5 \mathrm{ml}$ tubes. Next, cells were washed with PBS and centrifuged for $5 \mathrm{~min}$ at $200 \mathrm{~g}$. The supernatant was discarded and $1 \mathrm{ml}$ DNA staining solution added with $10 \mu \mathrm{l}$ permeabilization solution. Cells were subjected to vortex oscillation for 5-10 sec followed by incubation at room temperature under protection from light, and subsequently prepared for flow cytometric analysis.

Cells were resuspended in $500 \mu \mathrm{l}$ binding buffer after washing with PBS. Apoptosis analysis was performed using a FITC-AnnexinV/propidium iodide (PI) assay. Three groups were set as negative controls: i) Annexin-v-FITC was added to cells without PI; ii) PI was added to cells without Annexin-V-FITC and iii) neither Annexin-v-FITC nor PI was added to cells. Cells were incubated for $5 \mathrm{~min}$ under protection from the light after gentle vortex oscillation. The Annexin V-FITC/PI kit and PI were purchased from Hangzhou MultiSciences (Lianke) Biotech, Co., Ltd., (Hangzhou, China).

Sample preparation and label-free quantitative proteomic analysis. Cells were lysed using lysis buffer (4\% SDC in 0.1 M Tris-HCl, pH 8.0) with SigmaFast protease inhibitor (Sigma-Aldrich; Merck KGaA, Darmstadt, Germany). Equal amounts of protein from the four cell sublines were reduced with $10 \mathrm{mM}$ DTT and alkylated with $25 \mathrm{mM}$ iodoacetamide. An in solution digestion was carried out with sequencing grade-modified trypsin/Lys-C (Promega Corporation) at $37^{\circ} \mathrm{C}$ overnight. The peptides were acidated with a final concentration of $0.5-1 \%$ 
A

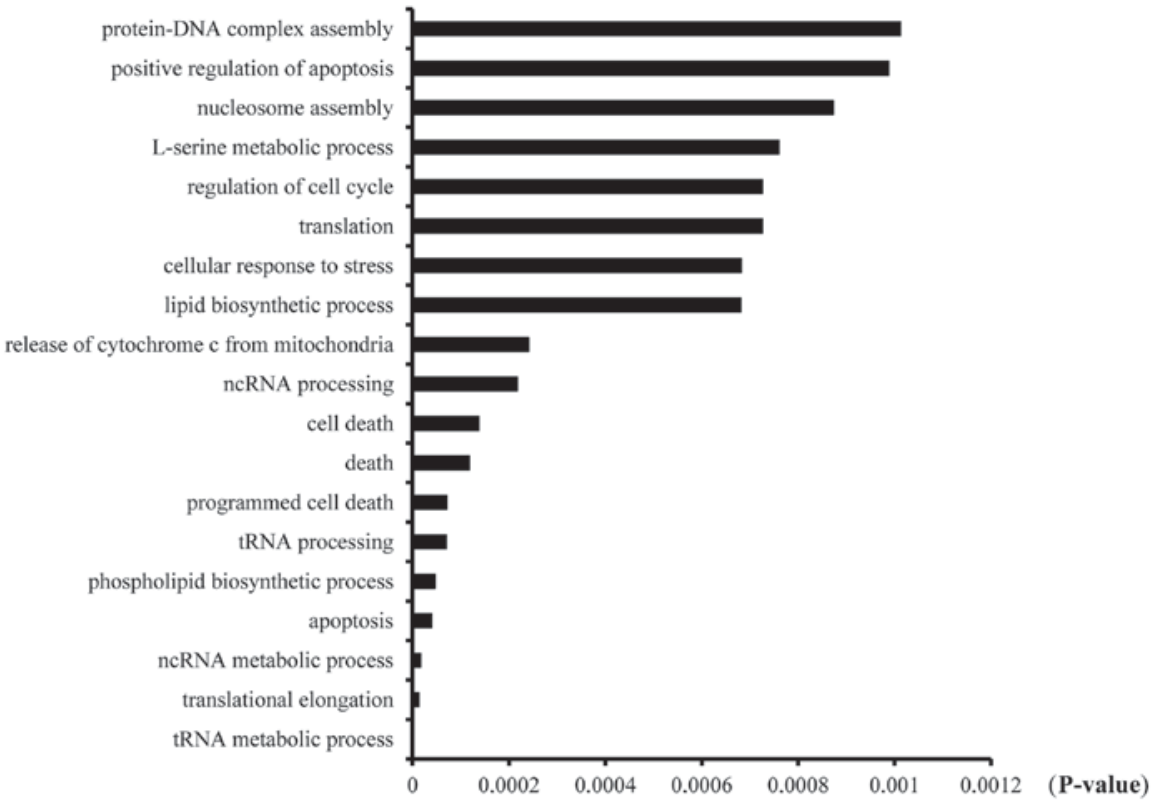

B

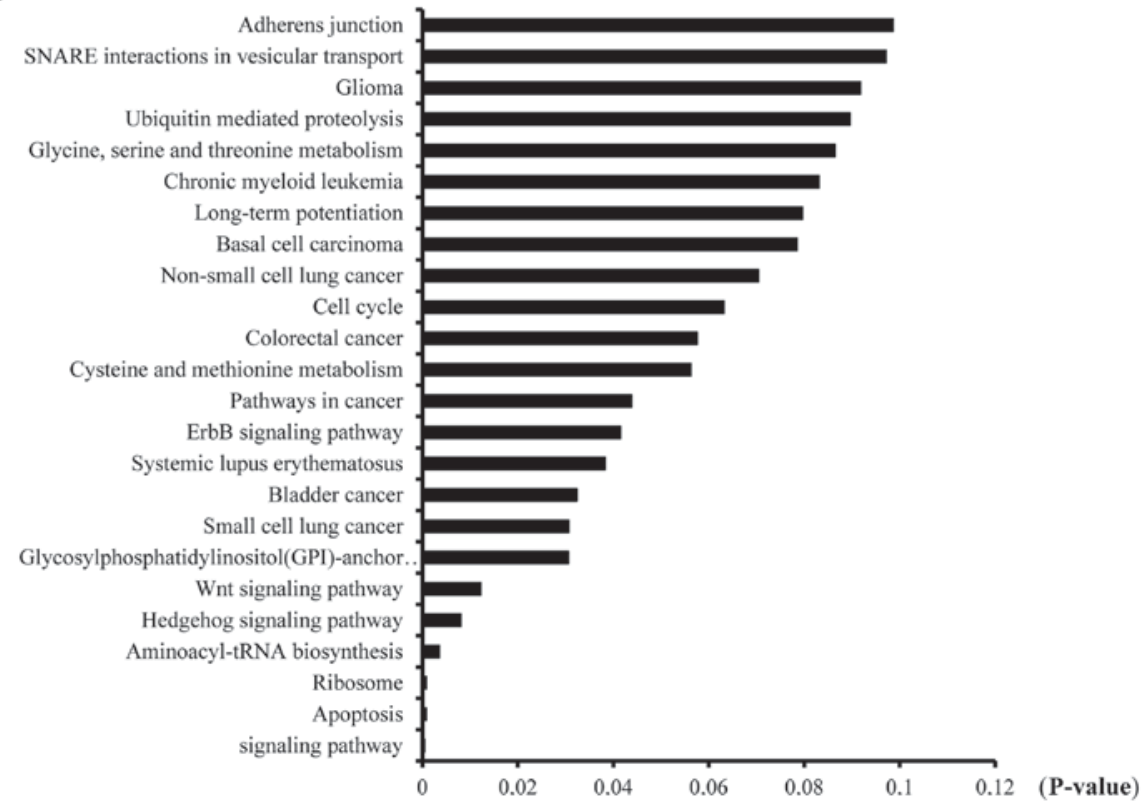

Figure 1. Bioinformatics analysis of differentially expressed mRNAs in cisplatin-sensitive (A2780) and -resistant (A2780-DR) cells. (A) KEGG analysis and (B) GO analysis using the DAVID bioinformatics platform (david.abcc.ncifcrf.gov/). KEGG, Kyoto Encyclopedia of Genes and Genomes; GO, Gene Ontology.

trifluoracetic acid (TFA) and SDC removed via high-speed centrifugation. Tryptic peptides were desalted, centrifuged in a speedvac for drying and redissolved in $0.1 \%$ FA.

For LC-MS/MS analysis, peptides were separated by a $90 \mathrm{~min}$ gradient elution at a flow rate of $0.20 \mu \mathrm{l} / \mathrm{min}$ on a Thermo Scientific EASY-nLC 1000 HPLC system directly interfaced with a Thermo Scientific Q Exactive mass spectrometer. The analytical column was a Thermo Scientific Acclaim RPepMap RSLC (50 $\mu \mathrm{m}$ ID, $15 \mathrm{~cm}$ length, C18, $2 \mu \mathrm{m}, 100 \AA$ ) and the precolumn was a Thermo Scientific AcclaimR PepMap100 column (100 $\mu \mathrm{m}$ ID, $2 \mathrm{~cm}$ length, C18, $5 \mu \mathrm{m}, 100 \AA$ A). Mobile phases A and B consisted of $0.1 \%$ formic acid and acetonitrile with $0.1 \%$ formic acid, respectively. A Q Exactive mass spectrometer was operated in data-dependent acquisition mode using Xcalibur 2.2 SP1 software and there was a single full-scan mass spectrum in the orbitrap (350-2,000 m/z, 70,000 resolution) followed by 15 data-dependent MS/MS scans at $27 \%$ normalized collision energy (HCD). MS/MS spectra from each LC-MS/MS run were searched against 'human. fasta' from UniProt (release date March 19, 2014; 68406 entries) using Proteome Discoverer (PD) software (v.PD1.4; Thermo Fisher Scientific, Inc.). In terms of search criteria, full tryptic specificity was required, two missed cleavages allowed, carbamidomethylation (C) set as the fixed modification, oxidation (M) set as the dynamic modification, precursor mass tolerances set at $10 \mathrm{ppm}$, and the fragment mass tolerance set at $0.02 \mathrm{Da}$. The peptide false discovery rate (FDR) was calculated using Percolator provided by PD. Relative protein quantification was performed using MaxQuant software (v.1.4.0.8). Quantitation was only carried out for proteins 
Table I. Different expressed genes identified by RNA-seq in cisplatin resistant and sensitivity ovarian cancer cell lines.

\begin{tabular}{lrcllcc}
\hline Gene & Value A & Value B & Ln (fold change) & A/B (fold change) & B/A (fold change) & P-value \\
\hline SELE & 156.81 & 0 & $-1.80 \times 10^{8}$ & Not valid & 0 & - \\
CAMK2D & 80.22 & 0 & $-1.80 \times 10^{8}$ & Not valid & 0 & - \\
ABCB1 & 12.65 & 0 & $-1.80 \times 10^{8}$ & Not valid & 0 & - \\
ARHGAP29 & 196.75 & 0.7 & -5.64 & 280.73 & $0.36 \times 10^{-2}$ & $2.47 \times 10^{-6}$ \\
SMARCA2 & 35.02 & 0 & $-1.80 \times 10^{8}$ & Not valid & 0 & - \\
NKAIN3 & 12.95 & 0 & $-1.80 \times 10^{8}$ & Not valid & 0 & - \\
\hline
\end{tabular}

Table II. Sequences of primers for reverse transcriptionquantitative polymerase chain reaction.

\begin{tabular}{ll}
\hline mRNA & \multicolumn{1}{c}{ Sequences $\left(5^{\prime}-3^{\prime}\right)$} \\
\hline SMARCA2F & CAGAAGATTGAGCAGGAGAGGAAAC \\
SMARCA2R & ATTGGCTACATACTCATCGGTCTGC \\
ACTBF & TGGCACCCAGCACAATGAA \\
ACTBR & CTAAGTCATAGTCCGCCTAGAAGCA \\
SELEF & AACACCCATCACCACTTCAATAG \\
SELER & CGAAACACTGTGAAGGGCAAAAGAA \\
CAMK2DF & TCTTGACAACTATGCTGGCTACA \\
CAMK2DR & TAGAATCGGTGAAAATCCATCCCTT \\
NKAIN3F & GACTGCCCTCTGGGTCACCTGGAA \\
NKAIN3R & CACATAACAGGCATACACAAAACCC \\
& ACC \\
ARHGAP29F & AAACTTCCACGAACACCATCCAG \\
ARHGAP29R & ACACTACAATGCCTTCACAATCC \\
ABCB1F & GTTCACTTCAGTTACCCATCTCG \\
ABCB1R & TATCGTGGTGGCAAACAATACAGG \\
\hline
\end{tabular}

with two or more unique peptide matches and PEP $<0.001$. Differentially expressed proteins were further confirmed by western blotting.

Western blot analysis. Proteins from cells were extracted in lysis buffer (Pierce; Thermo Fisher Scientific, Inc.) and a protease inhibitor cocktail (Sigma-Aldrich; Merck KGaA). Aliquots of protein samples $(20 \mu 1)$ were separated via SDS-PAGE. Samples were transferred onto PVDF membranes with a blotting system (ATTO) and membranes blocked with 5\% non-fat milk for $2 \mathrm{~h}$. Next, membranes were incubated overnight at $4^{\circ} \mathrm{C}$ with antibodies against SMARCA2, CAMK2D, cyclinE, Bcl-2, CDK4 and $\beta$-actin (1:1,000; ProteinTech Group, Inc., Chicago, IL, USA). After incubation with peroxidase-coupled anti-rabbit/mouse $\mathrm{IgG}$ (ProteinTech Group, Inc.) at $37^{\circ} \mathrm{C}$ for $2 \mathrm{~h}$, bound proteins were visualized with ECL (Pierce; Thermo Fisher Scientific, Inc.) and detected using a BioImaging System (UVP, Inc., Upland, CA, USA). Relative protein levels were quantified using $\beta$-actin as a loading control.

Statistical analysis. All experiments were performed in triplicate and data are presented as the mean \pm standard deviation. A nonparametric Student's t-test was used for the analysis of paired groups. All calculations were done with SPSS v.13.0 software (SPSS, Inc., Chicago, IL, USA). $\mathrm{P}<0.05$ was considered to indicate a statistically significant difference.

\section{Results}

mRNA expression profiles in cisplatin-sensitive and -resistant A2780 cells. Cisplatin resistance is one of the main problems encountered by cancer patients during cancer therapy. While numerous studies to date have identified genes associated with cisplatin resistance, the underlying mechanism remains unclear due to its complexity. To clarify the underlying pathways involved in the mechanism of cisplatin resistance development in ovarian cancer and identify contributory genes, we performed deep RNA sequencing, as described in previously, to compare the mRNA expression profiles between cisplatin-sensitive and -resistant A2780 cells (5). All RNA-seq data were analyzed using Bioinformatics technology (available upon request). In the present study, differentially expressed mRNAs between A2780 and A2780-DR cells were obtained from analyses using the DAVID bioinformatics platform (david.abcc.ncifcrf.gov/). GO and KEGG pathway analyses were included to further establish the biological relevance of differentially expressed mRNAs. The mRNAs showing significant alterations between A2780 and A2780-DR cells were classified into several groups of biological processes, including protein-DNA complex assembly (22; 1.038\%), positive regulation of apoptosis $(71 ; 3.35 \%)$, nucleosome assembly $(21 ; 0.991 \%)$, L-serine metabolic process $(6 ; 0.283 \%)$ and regulation of the cell cycle $(58 ; 2.738 \%$; Fig. 1A). Proteins encoded by the identified mRNAs were classified into several KEGG pathways, including adherens junction (15;0.708\%), SNARE interactions in vesicular transport $(9 ; 0.425 \%)$, glioma (13; 0/614\%), ubiquitin-mediated proteolysis $(24 ; 1.13 \%)$ and glycine, serine and threonine metabolism (8; 0.378\%; Fig. 1B).

Differentially expressed candidate genes in cisplatin-sensitive (A2780) and-resistant (A2780-DR) cells. To determine whether these genes were variably expressed in cisplatin-sensitive (A2780) and -resistant (A2780-DR) cells, six mRNAs were selected for RT-qPCR validation. As shown in Table I, some of these mRNAs were reported previously and others identified in the present study. Levels were quantified with a LightCycler 480 Real-Time PCR system and amplified with the aid of specifically designed primers (Table II). $\beta$-Actin was used for normalization and the relative levels calculated using the $\Delta \Delta \mathrm{Cq}$ method. The results showed higher expression levels of 

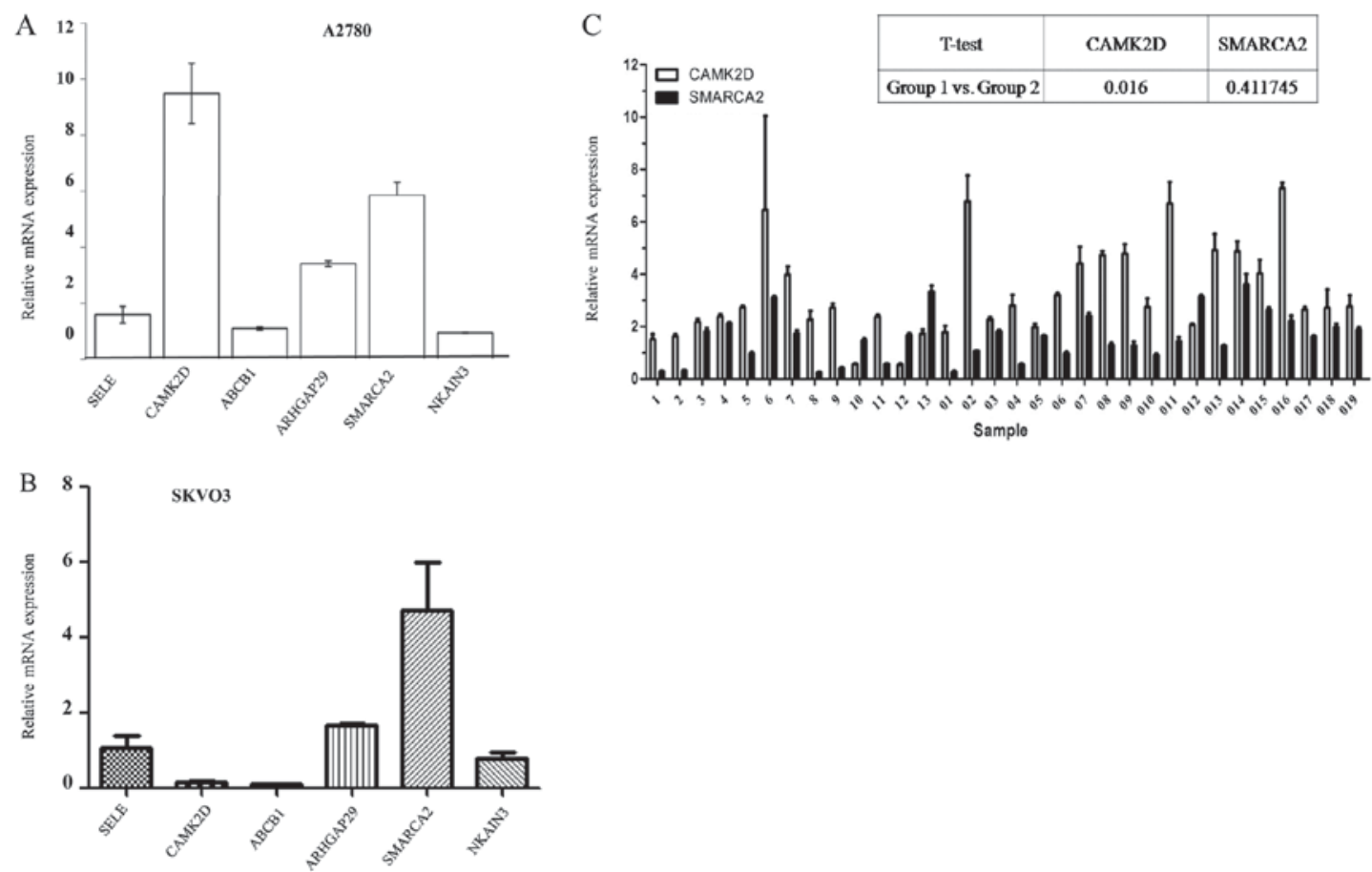

Figure 2. Validation of mRNA levels in different cell and tissue types. (A) ABCB1, CAMK2D, SMARCA2, ARHGAP29, SELE and NKAIN3 expression in cisplatin- sensitive A2780 and -resistant A2780-DR cells. Relative expression level was analyzed using the $2^{-\triangle A C q}$ method. (B) Relative expression of $A B C B 1$, CAMK2D, SMARCA2, ARHGAP29, SELE and NKAIN3 in SKVO3 cells with or without cisplatin treatment. (C) CAMK2D and SMARCA2 expression in samples of patients with different RFS rates. 1-13 represent patient samples with longer RFS and 01-019 represent patient samples with shorter RFS. cDNA hybrids of 32 cases were mixed as the control. RFS, recurrence free survival.

CAMK2D ARHGAP29 and SMARCA2 in cisplatin-resistant cells (A2780-DR), compared with sensitive cells (A2780), while the $A B C B 1$ and $N K A I N 3$ level was not (Fig. 2A). On the other hand, the expression levels of those genes in SKVO3 cells before and after cisplatin treatment didn't show the same pattern as in A2780 cells. SMARCA2 mRNA expression, but not that of $C A M K 2 D$, was significantly higher in SKVO3 cells after cisplatin treatment (Fig. 2B), indicating distinct roles of SMARCA2 and CAMK2D in different tumors. Based on this finding, we conducted further detailed studies on these two genes in association with cisplatin resistance development.

$C A M K 2 D$ is strongly associated with epithelial ovarian cancer (EOC) risk and SMARCA2 is frequently implicated as a tumor suppressor. To determine whether these two genes are putatively involved in cisplatin resistance, their mRNA levels in 32 selected patients with different RFS rates after hospital treatment were determined. Based on the RT-qPCR results, patients with shorter RFS (RFS $<12$ months, no. 01-019) had significantly higher SMARCA2 and CAMK2D levels, compared to those with longer RFS (RFS $>12$ months, no. 1-13; Fig. 2C). Our data indicate a higher risk of ovarian cancer recurrence in EOC patients with elevated $C A M K 2 D$ expression.

CAMK2D and SMARCA2 overexpression is associated with increased ovarian cancer cell survival under cisplatin treatment. To further verify whether SMARCA2 or CAMK2D influence cisplatin resistance, plasmids expressing either gene were transfected into A2780 cells and cisplatin sensitivity evaluated. After transfection, CAMK2D and SMARCA2 levels were evaluated via RT-qPCR. The viability of three cell lines (A2780, A2780-CAMK2D and A2780-SMARCA2) was measured after cisplatin treatment. The survival rate of A2780 cells was decreased to $<20 \%$ after treatment with $20 \mu \mathrm{M}$ cisplatin while that of A2780-CAMK2D cells was 38 and $30 \%$ for A2780-SMARCA2 cells (Fig. 3A and B).

$C A M K 2 D$ and $S M A R C A 2$ were additionally overexpressed in another ovarian cancer cell line, SKVO3, to validate their effects. The survival rate of cisplatin-treated cells increased from 20 to $25 \%$ in SKVO3-CAMK2D and $38 \%$ in SKVO3-SMARCA2 (Fig. 3C and D). Data from cell viability analyses in both A2780 and SKVO3 cell lines indicate that overexpression of either $C A M K 2 D$ or $S M A R C A 2$ increases cell resistance to cisplatin.

Conversely, knockdown of CAMK2D and SMARCA2 was performed in A2780-DR cells via siRNA transfection. Upon suppression of these genes, the survival rate of A2780-DR under cisplatin treatment was decreased, compared to that of control A2780-DR cells (Fig. 3E).

CAMK2D and SMARCA2 overexpression suppress apoptosis and influence the cell cycle under cisplatin treatment. Previous GO and KEGG pathway analyses suggest that CAMK2D and SMARCA2 are associated with regulation of the cell cycle and apoptosis. To establish the participation of these two genes in the above processes, A2780 cells were transfected with $C A M K 2 D$ or SMARCA2, harvested after cisplatin treatment and subjected to cell cycle and apoptosis analyses. Apoptosis analyses showed that overexpression of CAMK2D and SMARCA2 lead to a significant reduction in apoptosis of A2780 cells treated with cisplatin. Overall, $>20 \%$ A2780 cells underwent apoptosis after treatment, while 

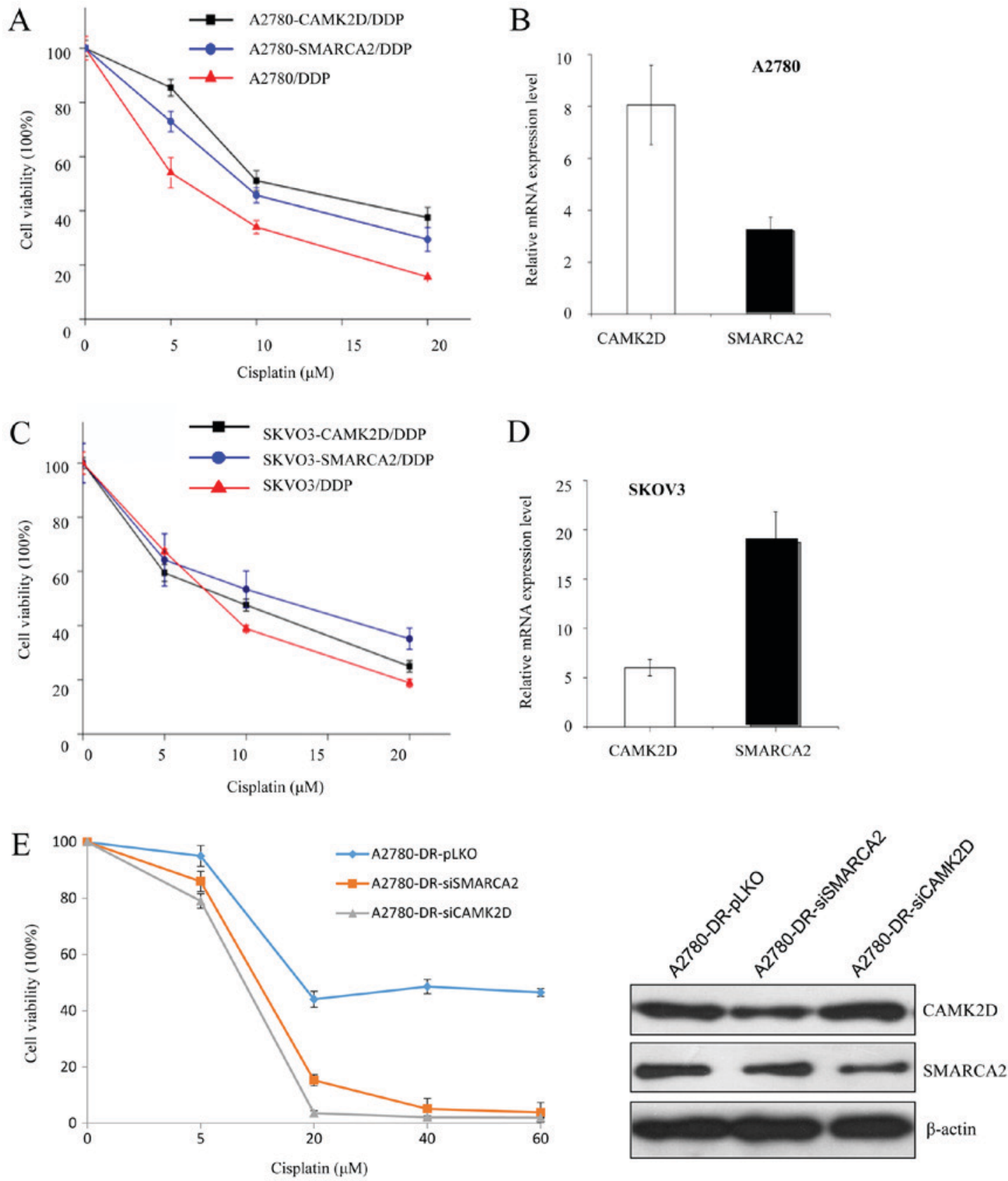

Figure 3. Survival rates of A2780 and SKVO3 cells overexpressing CAMK2D or SMARCA2 following DDP treatment. (A) Cell viability analysis of $C A M K 2 D$ and SMARCA2-overexpressong and control A2780 cells following DDP treatment $(0-20 \mu \mathrm{M})$ for $24 \mathrm{~h}$. (B) Relative CAMK2D and SMARCA2 mRNA expression in transfected A2780 cells. (C) Cell viability analysis of CAMK2D and SMARCA2-overexpressong and control SKVO3 cells following DDP treatment $(0-20 \mu \mathrm{M})$ for $24 \mathrm{~h}$. (D) Relative CAMK2D and SMARCA2 mRNA expression in transfected SKVO3 cells. (E) Cell survival rate of A2780-DR with SMARCA2 or CAMK2D knockdown. The SMARCA2 or CAMK2D level in A2780-DR cells was decreased by RNA interference. The protein level was evaluated by western blotting and the survival rate was compared between A2780-DR-pLKO and A2780-DR-siSMARCA2 (or A2780-DR-siCAMK2D) following various doses of DDP treatment. DDP, cisplatin.

only $\sim 10 \%$ apoptotic cells were detected in cells expressing CAMK2D or SMARCA2 (Fig. 4A). Similar results were obtained with the SKVO3 cell line, but the extent of apoptosis was lower than that in A2780/DDP (Fig. 4B). Our data suggest that $C A M K 2 D$ and SMARCA2 increase cancer cell resistance to cisplatin mainly via reducing apoptosis. Cell cycle analyses disclosed that after cisplatin treatment, the percentages of G1 and $\mathrm{G} 2 / \mathrm{M}$ cells were decreased while cells in the $\mathrm{S}$ phase were simultaneously increased, indicative of drug-induced defects in $\mathrm{G} 2 / \mathrm{M}$ progression. Upon $C A M K 2 D$ and SMARCA2 overexpression in A2780, the G2/M cell population was decreased $\sim 4 \%$ after cisplatin treatment while that in control A2780 cells was decreased $\sim 8 \%$, indicating a reduction in sensitivity to cisplatin-induced inhibition of cell proliferation (Fig. 5A). We additionally assessed the levels of CDK4, cyclin E and Bcl-2 related to the cell cycle and apoptosis, respectively, in the same sample group. Expression of these three proteins was higher in the A2780-CAMK2D and A2780-SMARCA2 cell lines than control A2780 cells (Fig. 5B).

Proteome profile comparison in different cell lines. To further elucidate the specific mechanisms by which $C A M K 2 D$ or 

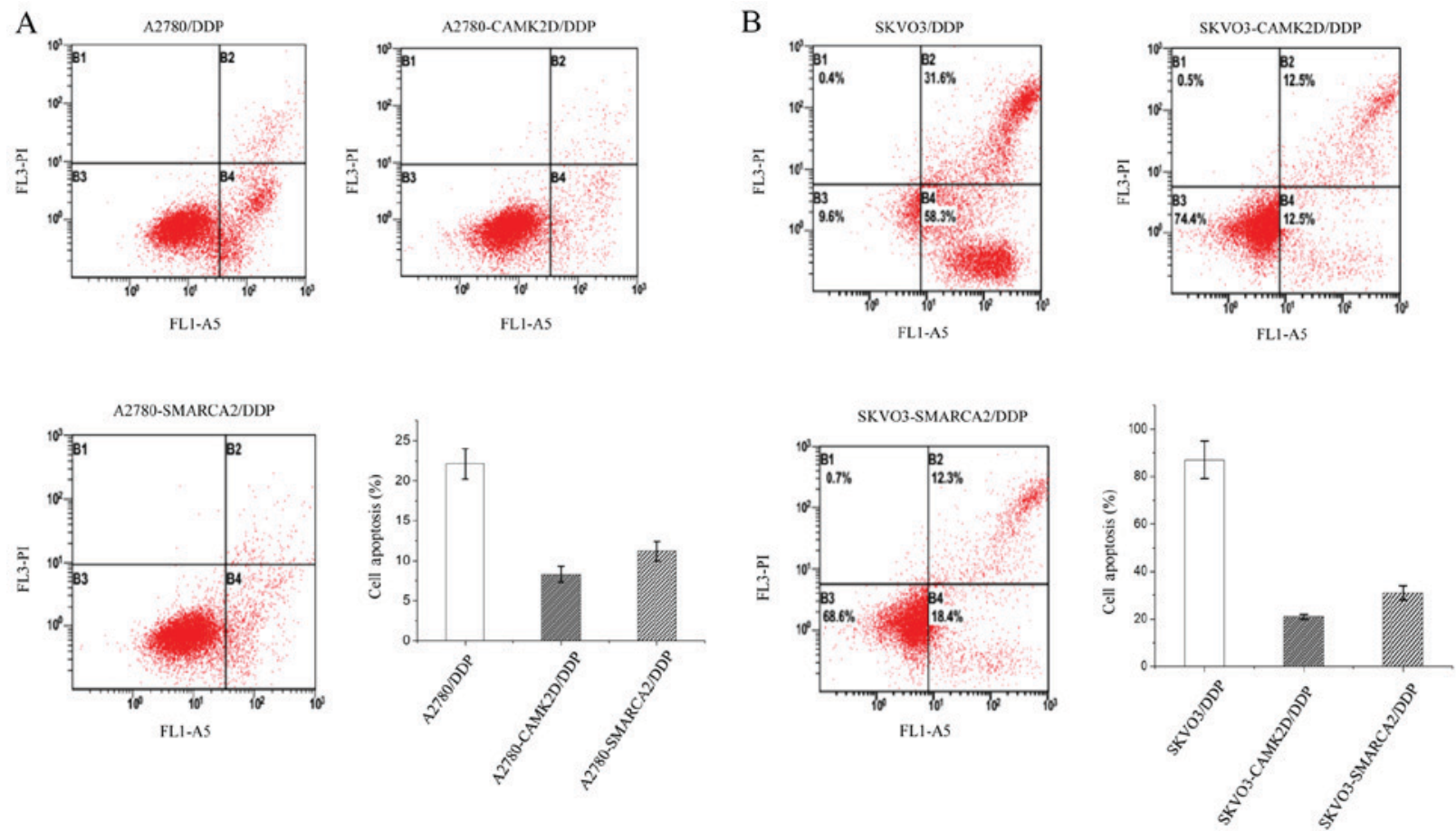

Figure 4. CAMK2D or SMARCA2 overexpression in A2780 or SKVO3 cells suppresses apoptosis. (A) Cellular apoptosis levels in different groups of A2780 cells following DDP treatment as detected using flow cytometry. (B) Cellular apoptosis levels in different groups of SKVO3 cells following DDP treatment as detected using flow cytometry. DDP, cisplatin.

A
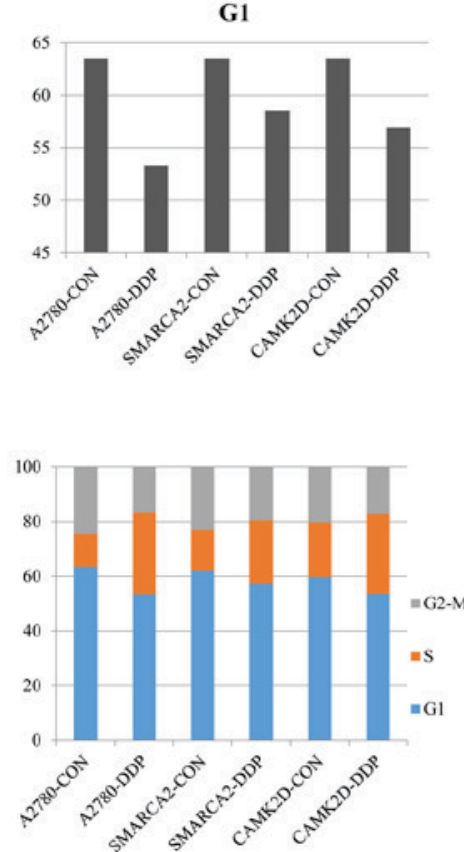

G2-M
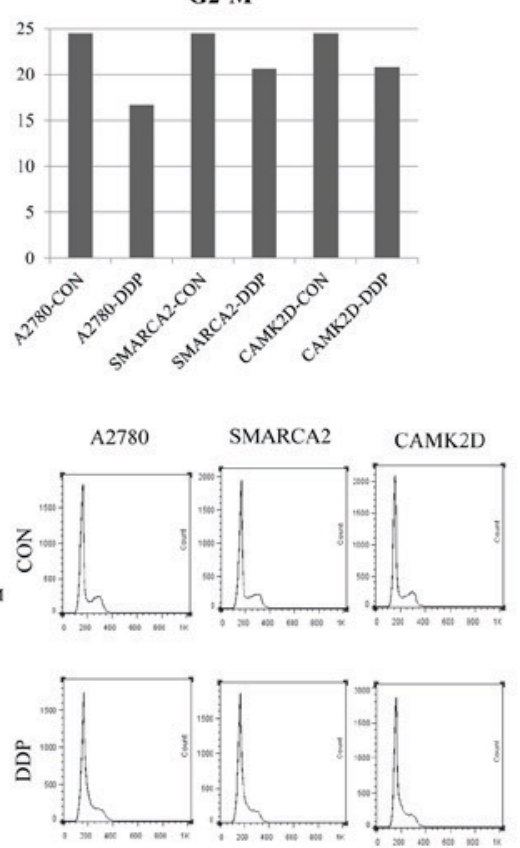

B
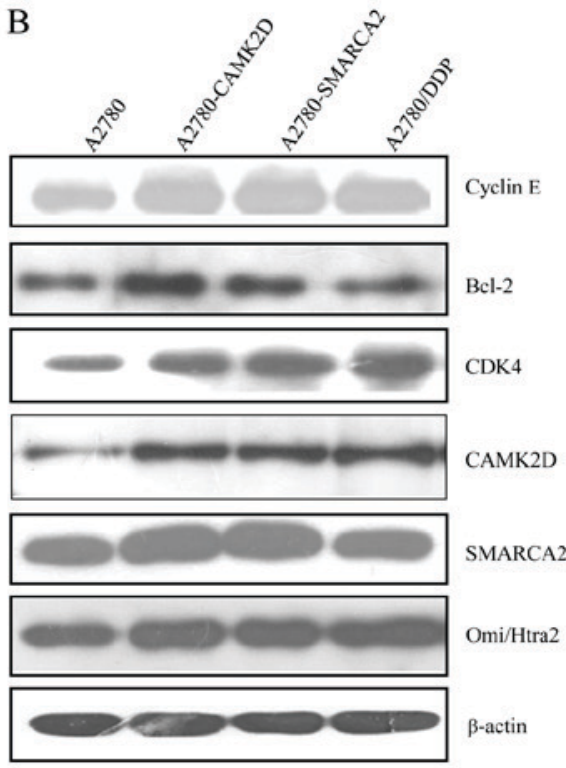

Figure 5. (A) Cell cycle distribution in different groups of A2780 cells following DDP treatment and (B) validation of SMARCA2, CAMK2D CyclinE, Bcl-2, CDK4 and Omi/Htra expression by western blot analysis. DDP, cisplatin; Bcl-2, B-cell lymphoma-2; CDK, cyclin dependent kinase.

SMARCA2 contributes to cisplatin resistance in ovarian cancer, quantitative proteomic analysis was conducted in A2780, A2780/DDP, A2780-SMACRA2 and A2780-CAMK2D cells. Protein expression changes after cisplatin treatment or SMARCA2 and CAMK2D overexpression were measured and change fold values of $>1.5$ or $<0.67$ selected for further analysis (available upon request). After cisplatin treatment, about 305 proteins (group I) involved in several processes of metabolism and disease development were highly expressed, 315 proteins (group II) displayed changes in expression after transfection with SMARCA2, and 250 (group III) were altered after transfection with $C A M K 2 D$. In total, 212 proteins were 


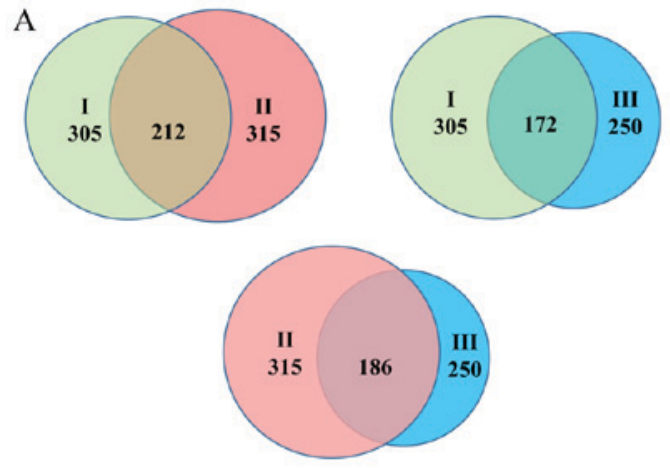

C

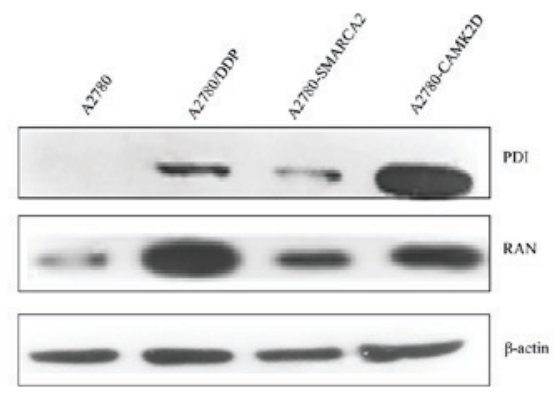

B

KEGG pathways
Spliceosome
Ribosome
RNAdegradation
Huntington's disease
Parkinson's disease
Amino sugar and nucleotide
sugar metabolism
Oxidative

phosphorylation

Butanoate metabolism

Pyrimidine metabolism

Alzheimer's disease

Fructoseandmannose

metabolism

Glycolysis / Gluconeogenesis

Purine metabolism

$\begin{array}{lll}\text { I } & \text { II } & \text { II } \\ \text { V } & \text { V } & \text { V } \\ \text { J } & & \text { V } \\ \sqrt{ } & \sqrt{ } & \sqrt{ } \\ \sqrt{ } & \sqrt{ } & \sqrt{ } \\ \sqrt{ } & \sqrt{ } & \sqrt{ } \\ \text { J } & & \end{array}$

III

$\checkmark$

$\sqrt{ }$

$\sqrt{ }$

$\sqrt{ }$

(n)

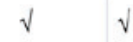

$\checkmark$

$\begin{array}{ll}\sqrt{ } & \sqrt{ } \\ & \sqrt{ }\end{array}$

$\checkmark$

Figure 6. Proteome profile comparison and analyses under different conditions. Protein expression changes following DDP treatment and SMARCA2 or CAMK2D overexpression. Change fold values $>1.5$ or $<0.67$ were selected for further analysis. (A) I, Differentially expressed proteins between A2780 (control) and A2780-DDP (cisplatin treatment); II, differentially expressed proteins between A2780 (control) and A2780-SMARCA2 (SMARCA2overexpression); III, differentially expressed proteins between A2780 (control) and A2780-CAMK2D (CAMK2D overexpression). (B) KEGG pathway analysis of differentially expressed proteins from I, II and III. (C) Validation of PDI and RAN protein levels in A2780 (control), A2780-DDP (DDP-treated), A2780-SMARCA2 (SMARCA2-overexpressing) and A2780-CAMK2D (CAMK2D-overexpressing) cells. DDP, cisplatin; KEGG, Kyoto Encyclopedia of Genes and Genomes.

altered in groups I and II, 172 proteins in groups I and III, and 186 proteins in groups II and III (Fig. 6A). Based on KEGG pathway analysis, the majority of these proteins contribute to spliceosome assembly, RNA degradation, oxidative phosphorylation, pyrimidine metabolism and the development of two neurodegenerative diseases (Huntington's and Parkinson's) (Fig. 6B). To further clarify the relationship between these proteins and $C A M K 2 D$ or $S M A R C A 2$ overexpression, we selected PDI and RAN and verified their expression patterns in A2780-CAMK2D, A2780-SMARCA2 and A2780 with or without DDP treatment (Fig. 6C). The signals for these two proteins in A2780-CAMK2D, A2780-SMARCA2 and A2780/DDP were significantly higher than those in A2780 cells. Further studies are warranted to ascertain how these proteins are involved in the development of cisplatin resistance of ovarian cancer and the underlying mechanisms.

\section{Discussion}

Drug resistance is a major problem in the effective treatment of cancer patients. Some cancer types are intrinsically resistant to chemotherapy while others develop drug resistance during treatment (20). To determine the overall changes in mRNA levels between cisplatin-sensitive and cisplatin-resistant ovarian cancer cells, we employed transcriptome sequencing and identified several candidate genes involved in resistance, many of which have been reported for the first time in this study. ABCB1, CAMK2D, SMARCA2, ARHGAP29, $S E L E$ and NKAIN3 were selected for quantitative RT-PCR analyses. Our data revealed relatively higher expression of $C A M K 2 D$ and SMARCA2 in cisplatin-resistant (A2780-DR) cells among the six candidates. Additionally, expression of CAMK2D was significantly different between tissue samples of patients from the longer and shorter RFS groups, supporting the potential importance of $C A M K 2 D$ and SMARCA2 in the development of drug resistance. To further ascertain the association of these two genes with cisplatin resistance, we transfected A2780 and SKOV3 cells with $C A M K 2 D$ and SMARCA2 overexpression plasmids and evaluated the viability of cells under cisplatin treatment. In both cell types, CAMK2D and SMARCA2 overexpression led to increased cell viability indicative of resistance to cisplatin, compared with the control groups.

A variety of drugs, such as cisplatin, paclitaxel, etoposide and vinblastine, kill tumors through inducing apoptosis (21). Accordingly, we performed flow cytometry analysis to determine whether $C A M K 2 D$ and SMARCA2 influence drug resistance through effects on the apoptotic mechanism. Notably, following overexpression of these two genes, the percentage of apoptotic cells in both A2780 and SKVO3 cell lines was significantly decreased. Additionally, protein expression of Bcl-2, one of the most important cancer genes related to apoptosis, was markedly upregulated, supporting the theory that $C A M K 2 D$ and $S M A R C A 2$ promote ovarian cancer cell resistance to cisplatin by reducing apoptosis. SMARCA2 and CAMK2D protein levels were affected by each other, indicating a potential reciprocal association. To elucidate the mechanisms by which CAMK2D and SMARCA2 
contribute to drug resistance, we further performed quantitative proteomic analysis to identify the potential proteins regulated by either gene. Bioinformatics analyses showed that SMARCA2 and CAMK2D induce similar pathways as cisplatin that contribute significantly to cellular metabolism and disease development. Notably, CAMK2D affects more pathways than SMARCA2, suggesting a more complex relationship with drug resistance progression. The potential association between $C A M K 2 D$ and SMARCA2 and their involvement in the development of drug resistance should be investigated in detail in future studies.

\section{Acknowledgements}

Not applicable.

\section{Funding}

The present study was supported by grants from the National Basic Research Program of China (grant no. 2015CB910600), the National Natural Science Foundation of China (grant nos. 31210103904 and 31700688), the Natural Science Foundation of Zhejiang Province (grant no. LY16C050003), the third level of the Zhejiang Province '151 talents project' (for Zhiguo Zheng) and the Zhejiang Cancer Hospital Biospecimen Repository and National Human Genetic Resources Sharing Service Platform (grant no. 2005DKA21300).

\section{Availability of data and materials}

The datasets used and/or analyzed during the current study are available from the corresponding author on reasonable request.

\section{Authors' contributions}

YJH and HX designed and directed the project. XLX and ZGZ performed the experiments. LLJ and SSS performed the RT-qPCR for each cell line and patient samples. QQT, TNS and BWL performed the bioinformatics analysis. YJH, ZGZ and $\mathrm{HX}$ wrote the paper and all authors participated in editing it.

\section{Ethics approval and consent to participate}

The present study was approved by the Zhejiang Cancer Hospital Ethics Committee (2012-03-7) and informed consent was obtained from all participants prior to their inclusion.

\section{Patient consent for publication}

Not applicable.

\section{Competing interests}

The authors declare that they have no competing interests.

\section{References}

1. Wu H, Wang K, Liu W and Hao Q: Recombinant adenovirus-mediated overexpression of PTEN and KRT10 improves cisplatin resistance of ovarian cancer in vitro and in vivo. Genet Mol Res 14: 6591-6597, 2015.
2. Schumann C, Chan S, Khalimonchuk O, et al: Mechanistic nanotherapeutic approach based on siRNA-mediated DJ-1 protein suppression for platinum-resistant ovarian cancer. Mol Pharm 13: 2070-2083, 2016.

3. Duan Z BK and Seiden MV: Inhibition of ABCB1 (MDR1) and ABCB4 (MDR3) expression by small interfering RNA and reversal of paclitaxel resistance in human ovarian cancer cells. Mol Cancer Ther 3: 833-838, 2004.

4. Ren L, Xiao L and Hu J: MDR1 and MDR3 genes and drug resistance to cisplatin of ovarian cancer cells. J Huazhong Univ Sci Technolog Med Sci 27: 721-724, 2007.

5. Zheng ZG, Xu H, Suo SS, Xu XL, Ni MW, Gu LH, Chen W, Wang LY, Zhao Y, Tian B and Hua YJ: The essential role of H19 contributing to cisplatin resistance by regulating glutathione metabolism in high-grade serous ovarian cancer. Sci Rep 6: 26093, 2016.

6. Jin AH and WeiZL: Molecular mechanism of increased sensitivity of cisplatin to ovarian cancer by inhibition of microRNA-23a expression. Int J Clin Exp Med 8: 13329-13334, 2015.

7. Kim DK, Seo EJ, Choi EJ, Lee SI, Kwon YW, Jang IH, Kim SC, Kim KH, Suh DS, Seong-Jang K, et al: Crucial role of HMGA1 in the self-renewal and drug resistance of ovarian cancer stem cells. Exp Mol Med 48: e255, 2016.

8. Liu FT, Pan H, Xia GF, Qiu C and Zhu ZM: Prognostic and clinicopathological significance of long noncoding RNA H19 overexpression in human solid tumors: Evidence from a meta-analysis. Oncotarget 7: 83177-83166, 2016.

9. Sun KX, Jiao JW, Chen S, Liu BL and Zhao Y: MicroRNA-186 induces sensitivity of ovarian cancer cells to paclitaxel and cisplatin by targeting ABCB1. J Ovarian Res 8: 80, 2015.

10. Zeng Q, Tao X, Huang F, Wu T, Wang J, Jiang X, Kuang Z and Cheng B: Overexpression of miR-155 promotes the proliferation and invasion of oral squamous carcinoma cells by regulating BCL6/cyclin D2. Int J Mol Med 37: 1274-1280, 2016.

11. Xie C, Han Y, Fu L, Li Q, Qiu X and Wang E: Overexpression of CARMA3 is associated with advanced tumor stage, cell cycle progression and cisplatin resistance in human epithelial ovarian cancer. Tumour Biol 35: 7957-7964, 2014.

12. Gao M, Miao L, Liu M, Li C, Yu C, Yan H, Yin Y, Wang Y, Qi X and Ren J: miR-145 sensitizes breast cancer to doxorubicin by targeting multidrug resistance-associated protein-1. Oncotarget 13: 59714-59726, 2016.

13. Permuth-Wey J, Chen YA, Tsai YY, Chen Z, Qu X, Lancaster JM, Stockwell H, Dagne G, Iversen E, Risch H, et al: Inherited variants in mitochondrial biogenesis genes may influence epithelial ovarian cancer risk. Cancer Epidemiol Biomarkers Prev 20: 1131-1145, 2011.

14. Wu Q, Madany P, Akech J, Dobson JR, Douthwright S, Browne G, Colby JL, Winter GE, Bradner JE, Pratap J, et al: The SWI/SNF ATPases are required for triple negative breast cancer cell proliferation. J Cell Physiol 230: 2683-2694, 2015.

15. Saddouk FZ, Sun LY, Liu YF, Jiang M, Singer DV, Backs J, Van Riper D, Ginnan R, Schwarz JJ, Singer HA: Ca2+/calmodulin-dependent protein kinase II- $\gamma$ (CaMKII $\gamma$ ) negatively regulates vascular smooth muscle cell proliferation and vascular remodeling. FASEB J 30: 1051-1064, 2016.

16. Tang S, Pan Y, Wang Y, Hu L, Cao S, Chu M, Dai J, Shu Y, Xu L, Chen J, et al: Genome-wide association study of survival in early-stage non-small cell lung cancer. Ann Surg Oncol 22: 630-635, 2015.

17. Wilson BG, Helming KC, Wang X, Kim Y, Vazquez F, Jagani Z, Hahn WC and Roberts CW: Residual complexes containing SMARCA2 (BRM) underlie the oncogenic drive of SMARCA4 (BRG1) mutation. Mol Cell Biol 34: 1136-1144, 2014.

18. Hoffman GR RR, Buxton F, Xiang K, McAllister G and Frias E: Functional epigenetics approach identifies BRM/SMARCA2 as a critical synthetic lethal target in BRG1-deficient cancers. Proc Natl Acad Sci USA 111: 3128-3133, 2014.

19. Puvanenthiran S, Essapen S, Seddon AM and Modjtahedi H: Impact of the putative cancer stem cell markers and growth factor receptor expression on the sensitivity of ovarian cancer cells to treatment with various forms of small molecule tyrosine kinase inhibitors and cytotoxic drugs. Int J Oncol 49: 1825-1838, 2016.

20. Ho AS, Kannan K, Roy DM, Morris LG, Ganly I, Katabi N, Ramaswami D, Walsh LA, Eng S, Huse JT, et al: The mutational landscape of adenoid cystic carcinoma. Nat Genet 45: 791-798, 2013.

21. Chang H, Sheng JJ,Zhang L, Yue ZJ,Jiao B, Li JS, et al: ROS-induced nuclear translocation of calpain-2 facilitates cardiomyocyte apoptosis in tail-suspended rats. J Cell Biochem 116: 2258-2269, 2015. 